\title{
Direct Observation of Localized Modes in an Open Disordered Microwave Cavity
}

\author{
F. Mortessagne, D. Laurent, O. Legrand, P. Sebbah \\ and Ch. VAnneste \\ Laboratoire de Physique de la Matière Condensée, CNRS UMR 6622 \\ Université de Nice Sophia-Antipolis, 06108 Nice, France
}

\begin{abstract}
We present measurements of the spatial intensity distribution of localized modes in a two-dimensional open microwave cavity randomly filled with cylindrical dielectric scatterers. The robustness of these modes with respect to a modification of the boundaries of the scattering region is successfully established. From a comparison with numerically calculated modes, we can extract precise values of the localization length which are in agreement with the measured spectral widths. These results constitute unambiguous signatures of the existence of strongly localized electromagnetic modes in two-dimensional open random media.
\end{abstract}

PACS numbers: 42.25.Dd, 72.15.Rn

\section{Introduction}

In a strongly disordered scattering medium, electronic or classical waves can build spatially localized modes (with exponentially decaying envelope). As a consequence, unusual transport properties are expected due to a renormalization of the diffusion coefficient [1-4]. In spite of decades of intensive research, experimental observation of strong localization of classical waves remains a tremendous challenge (see Ref. [5] for a review concerning photons). One of the main difficulties lies in the fact that the signature of localization is sought after in the exponential decrease in transmission [6]. Still, another unavoidable phenomenon manifests itself through an exponential decrease, namely absorption [7]. This effect remains a strong hindrance even though Störzer et al. have observed, in a recent work, deviations from diffusion which cannot be explained by absorption but plead for a transition to strong localization of photons in three dimensions [8]. Absorption was already discriminated in the original approach proposed by Weaver through transient analysis of ultrasound measurement in a two-dimensional (2D) disordered medium [9]. Another way to seek for localization has been proposed 
by Genack and co-workers who developed a statistical approach of localization in quasi one-dimensional (1D) disordered microwave systems [10]. In all these works, the localization is analyzed in terms of transport but little attention is paid to individual localized modes which are the building blocks of this phenomenon (except for recent experiments in 1D systems [11]). In 2D microwave cavities, a few authors have tried to provide evidence of such localized modes [12]. However, all their experimental systems present the same limitation: every shown mode is clearly influenced by the reflecting boundaries of the cavity, thereby precluding the unambiguous conclusion of attributing the localization effect to the sole presence of the scatterers. When one wishes to provide evidence for a strong coherent interference effect through multiple scattering one should avoid trivial trapping due to boundaries. Indeed, a spatial concentration of the field near the limits of the cavity does not prove the existence of a localized mode unambiguously. The aim of this paper is thus to propose a direct observation of localized modes in an open disordered 2D microwave cavity. Beyond the mere observation of such modes, we will put them under a test of robustness and successfully confront them to numerical simulations and theoretical predictions.

\section{Experimental}

All our results are obtained from transmission signals measured in a 2D microwave cavity operated at frequencies ranging from $500 \mathrm{MHz}$ to $10 \mathrm{GHz}$. The original bare cavity is composed of two copper plates sandwiching a copper rectangular frame of thickness $5 \mathrm{~mm}$. To reduce ohmic losses we used oxygen free hard copper (OFHC). Due to its height of $5 \mathrm{~mm}$ (smaller than half the smallest wavelength used), this cavity only admits transverse magnetic two-dimensional modes of order 0 . Through one of the copper plates, a few antennas are introduced, their optimal coupling being obtained by fixing their penetration length inside the cavity at $2 \mathrm{~mm}$. The antennas are monopolar with SMA connectors and their positions are displayed in Fig. 1. In transmission measurements, only two antennas are used at a time, the other ones being terminated by $50 \Omega$ loads so that all antennas behave in the same way regarding the losses they imply. These antennas are linked to an HP 8720 D vector analyzer through flexible cables. The details about transmission measurements are described in Ref. [13].

In order to mimic a truly open system, frames of microwave absorbing foams are inserted in the cavity, surrounding an empty rectangular-shaped space. Two different layers of absorbers (ECCOSORB® LS-14 and LS-16) are used with different electromagnetic impedances (see Fig. 1). Their thickness is the overall thickness of the cavity and their widths are determined to provide $20 \mathrm{~dB}$ of attenuation in the intensity reflected back to the empty space at frequencies above $500 \mathrm{MHz}$. As shown in Fig. 1 a disordered medium is introduced in the empty space. It consists of 196 cylindrical dielectric scatterers with a measured dielectric constant $\epsilon=37$, a radius of $3 \mathrm{~mm}$ and a height of $5 \mathrm{~mm}$. These scatterers have 


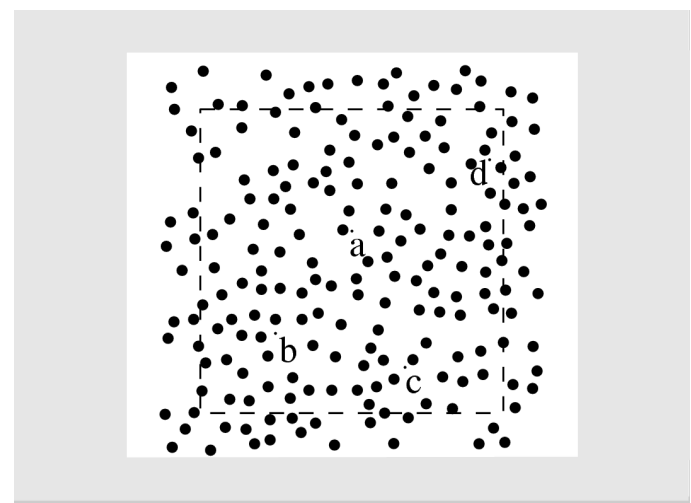

Fig. 1. Schematic view of the cavity: 196 scatterers located in a central square region. For some measurements, the scatterers outside the dashed square are withdrawn. Two different layers of microwave absorbers ensure a weak inward-reflection of the field. The antennae $(a, b, c, d)$ are shown through small dots.

been chosen for their particular low loss (quality factor $Q=7000$ at $7 \mathrm{GHz}$ ) and their high index. In the frequency range of interest, the possible excitation of transverse electric waves inside the dielectric scatterers can be assumed negligible.

\section{Results and discussion}

The positions of the scatterers are determined at random with a filling fraction of $8.9 \%$ and a minimal distance $d_{\min }$ between the centers of neighboring scatterers. At the above given filling fraction, the values of $d_{\min }$ range from $8 \mathrm{~mm}$ to $19 \mathrm{~mm}$. The smallest value is dictated by practical constraint, the largest corresponds to the lattice parameter of a square array. We fixed $d_{\min }=11 \mathrm{~mm}$. Figure 1 displays a particular realization of disorder using the above parameters. In Fig. 2, a typical transmission (between antennas $a$ and $b$ ) spectrum is shown. One can clearly identify a frequency range which is reminiscent of the band gap found in the case of the periodic structure with the same filling fraction. This gap is strongly correlated with the Mie cross-section for a single scatterer [14] (the Mie cross-section is shown superimposed to the measured spectrum in Fig. 2). Using a diffusion based theory [15-17] we evaluated the localization length $\xi_{\text {theory }}$ in two dimensions for our system of scatterers within the independent scattering approximation (ISA). It reads

$$
\xi_{\text {theory }}=\ell \exp \left(\pi \Re\left(k_{\text {eff }}\right) \ell / 2\right),
$$

where $k_{\text {eff }}$ is the effective complex wave number and $\ell=\left(2 \Im\left(k_{\text {eff }}\right)\right)^{-1}$ is the mean free path, which is inversely proportional to the Mie cross-section. The minimal values of this localization length are roughly located at the maxima of the Mie cross-section and are of the order of $10 \mathrm{~mm}$, a value comparable to the mean free path and also to the mean distance between scatterers for the filling fraction 
mentioned above. We are well aware of the fact that the ISA is not a valid approximation in the regime we investigate in the present letter and that a collective effect, similar in nature to the proximity resonance effect [18], tends to produce extra sharp resonances which are blue-shifted with respect to the Mie resonances. Let us note that Sebbah and Vanneste [19], in a numerical study of random lasing in the localized regime, also noticed that the frequencies of the localized modes lie between the maxima of the Mie cross-section. This is also clearly confirmed in our findings. However, in spite of these important drawbacks, as will be established later, the ISA based theory has the principal merit of yielding the right orders of magnitude for the minimal values of the localization length.

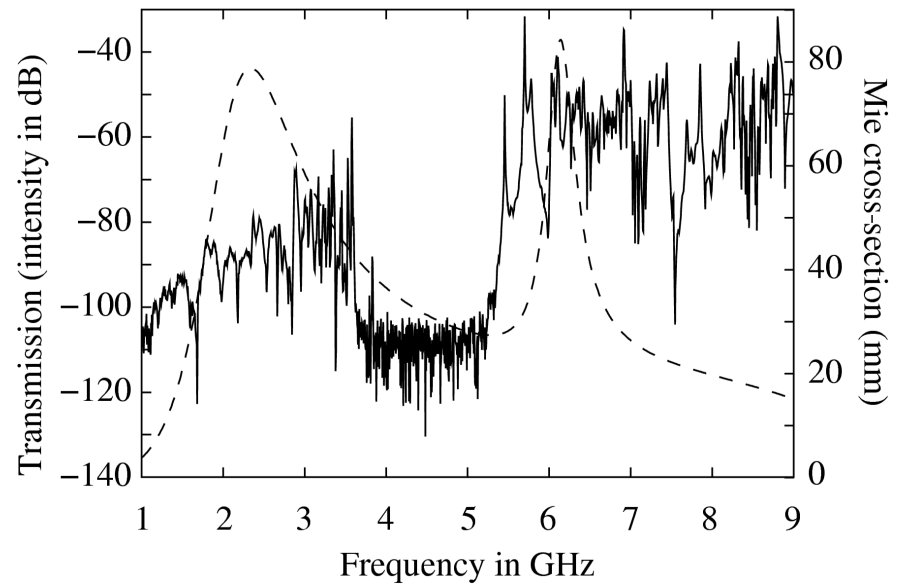

Fig. 2. Full curve: transmission signal measured for the disordered realization shown in Fig. 1 (log scale). Dashed curve: Mie cross-section of a single dielectric scatterer.

By using an appropriate fitting procedure [20] we extracted the spectral widths of the resonances. Only a few widths take values close to those expected taking only the ohmic losses into account [13], whereas the vast majority are significantly larger. These purely ohmic widths could be associated to resonances which very likely do not reach the absorbing foam at the boundary. Localized modes, if any, should be found among these resonances. We focused our attention on three of them: one in the gap at $5.45 \mathrm{GHz}$, one on the edge of the gap at $5.66 \mathrm{GHz}$, and one at $7.80 \mathrm{GHz}$. For different couples of antennas we obtained spectra very similar to the one presented in Fig. 2, the most sensitive resonances being the sharpest, like the three selected, as they can even disappear for a given couple. This behavior pleads in favor of the observation of truly localized modes.

We obtained the spatial distribution of these resonances by using a well-known scanning perturbation technique (see Ref. [21] and references therein). This technique is based on the fact that, for a given resonance, a frequency shift is caused by the presence of a small stainless steel bead placed inside the cavity. 


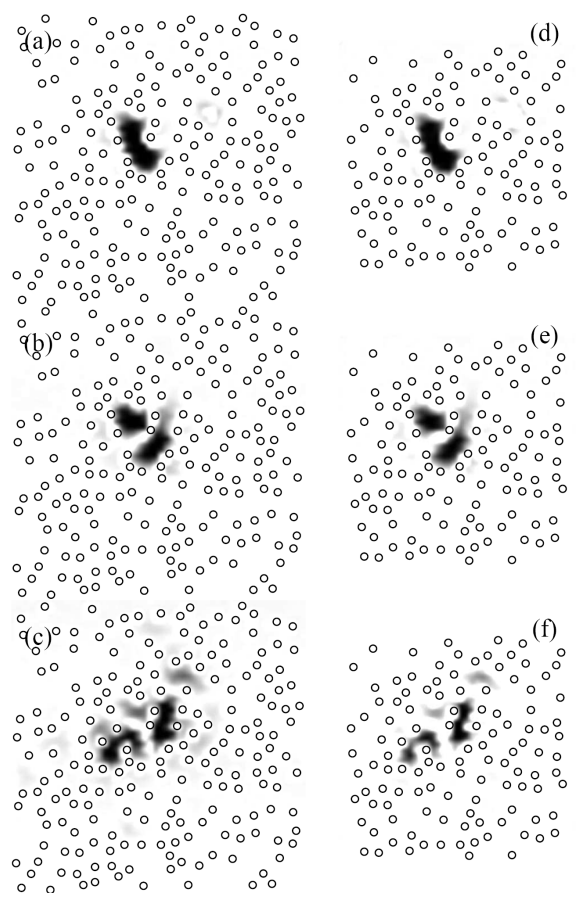

Fig. 3. Experimental maps of the amplitude of the electric field for localized modes at $5.45 \mathrm{GHz}$ (a) and (d), $5.66 \mathrm{GHz}(\mathrm{b})$ and (e), and at $7.80 \mathrm{GHz}$ (c) and (f), for two different sizes of the scattering region (a $25 \mathrm{~cm} \times 25 \mathrm{~cm}$ square and a $20 \mathrm{~cm} \times 20 \mathrm{~cm}$ square). The 3 modes are clearly very little sensitive to the suppression of the peripheral scatterers.

This shift is a measure of the intensity of the local field at the bead location. More precisely, this shift can be shown to be essentially proportional to the square of the electric field [21]. Indeed, through measurements on computable modes of a rectangular cavity we could check that the transverse magnetic field contribution is at most of the order of $1 \%$ of the maximum contribution of the electric field. The bead can be moved on a very finely defined grid ( $5 \mathrm{~mm}$ step) from the outside of the cavity by means of a strong magnet which is fixed on an $X-Y$ precise translation stage.

In Fig. $3 \mathrm{a}-\mathrm{c}$ two-dimensional scans of the amplitude (square root of the intensity) of the three long-lived modes mentioned above are depicted. Spatial localization is obvious! Whereas mode (a) seems to be trapped in a small region surrounded by scatterers, modes (b) and (c) are clearly more extended. Following a method indicated below, we could evaluate the localization lengths for these modes: between 15 and $25 \mathrm{~mm}$ for mode (a), 25-36 mm for mode (b) and 38-50 $\mathrm{mm}$ for mode (c) (the ranges depending on the anisotropy of the spatial pattern). 


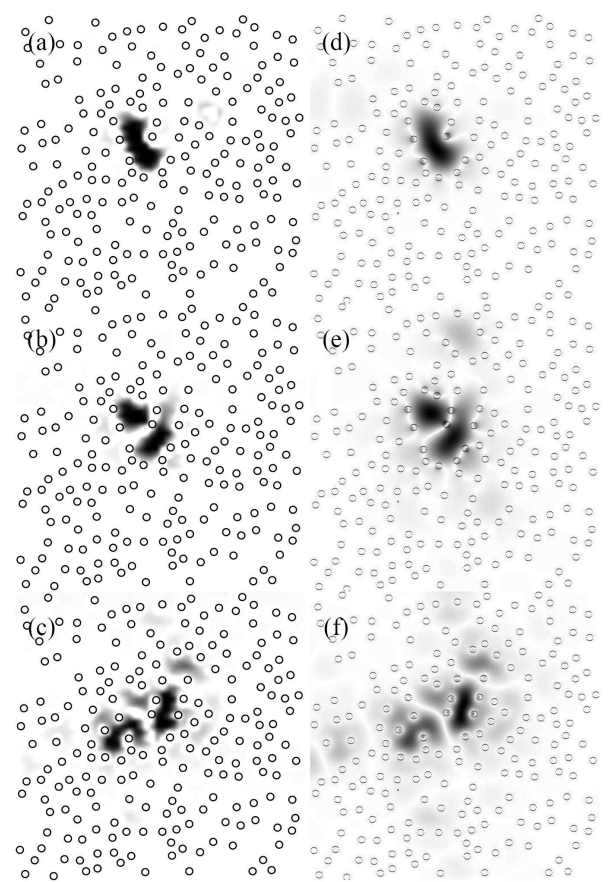

Fig. 4. Comparison between experimental results (a), (b), and (c) and numerical FDTD-based simulations (with perfectly open boundary conditions) (d), (e), and (f); the agreement is excellent.

In order to test the robustness of these modes with respect to a modification of the boundaries of the scattering region, we reduced this region from a $25 \mathrm{~cm}$ $\times 25 \mathrm{~cm}$ square to a $20 \mathrm{~cm} \times 20 \mathrm{~cm}$ square. This was simply achieved by taking away the outer scatterers as shown in Fig. 3d-f. The frequency spectrum we obtained is globally similar to the previous one shown in Fig. 2 but certainly not superimposable. But the important point is that the peaks associated to the 3 modes shown above are still there with very little perturbed central frequencies and comparable sharpness. The two-dimensional scans of the amplitude of the corresponding modes are shown as (d), (e), (f) in Fig. 3. Obviously, the important modification of the boundaries of the scattering region did not affect the integrity of these modes that we can most legitimately qualify as localized.

As our scanning technique allows only a limited sensitivity for small electric field amplitudes (due to a small contribution from the squared magnetic field to the frequency shift), we also performed numerical simulations of the Maxwell equations through a finite-difference time-domain (FDTD) method [22]. To mimic perfectly open boundary conditions perfectly matched layer (PML) boundaries have been implemented [23]. These PML conditions are used in the same spirit as the absorbing microwave layers of the actual experiment. In the numerical simu- 
lations, losses are only due to the leakage through the boundary since no ohmic dissipation is included. Hence, spectral responses display sharper peaks than in the experiment but all the sharp resonances of the experiment are found at the corresponding frequencies. Figure 4 shows a comparison between experimental and simulated modes. The agreement is spectacular. Let us note the presence of a nonvanishing field inside the scatterers, such information being beyond the reach of our experimental measuring technique. As the calculated modes have precisely calculated exponentially vanishing tails far from their centers, we could extract values of their localization lengths $\xi_{\text {loc }}$ by numerically evaluating the field amplitude autocorrelation. The values of $\xi_{\text {loc }}$ thus obtained are in complete agreement with the minimal values of $\xi_{\text {theory }}$. Moreover, the observed spectral widths $\Gamma_{\text {leak }}$ due to leaking at the boundary, are also in fair agreement with the gross evaluation given by $\Gamma_{\text {leak }} \simeq \exp \left(-2 R / \xi_{\text {loc }}\right)$ with $R$ the distance from the center of the mode to the boundary [24]. More specifically, when reducing the scattering region, hence reducing $R$, we were able to ascertain the above relationship by extracting values of $\Gamma_{\text {leak }}$ from the total experimental widths thanks to a proper evaluation of the contribution of ohmic losses to the latter [13]. All this confirms that the exponentially vanishing field of such modes at the boundary involves all the scatterers of the system through complex multiple interference effects. In consequence, we can conclude that the modes we observe should not be simply considered as defect-like modes or cavity modes built by the mere presence of a few scatterers surrounding them. Thus, in spite of their high refraction index, all our results show that the scatterers we use should not be viewed as simple walls at the frequencies of the localized modes but as true resonators. This is numerically clearly illustrated by the presence of field maxima inside certain scatterers (see Fig. 4). To be complete, other realizations of the same type of disorder with the same scatterers have been studied and all the localized modes that were measured confirm the above results. This will be related at length in a forthcoming publication.

\section{Summary}

In summary, we have provided strong evidences in favor of the first observation of truly localized modes in a two-dimensional open disordered microwave cavity. Most importantly, we have clearly shown how these localized modes are robust regarding strong modifications of the boundary of the scattering region. The fact that the non-ohmic parts of the measured modal widths vary in inverse proportion of the exponential of the distance of these modes to the boundary also provides a strong argument in favor of strong localization in finite-size open systems and the way to probe it efficiently via measurements of decay rates.

\section{Acknowledgments}

We are very grateful to Gregory Sauder for helping us with the automatization of the data acquisition. D.L. acknowledges financial support from DGA/CNRS grant no. 2004487. 


\section{References}

[1] P.W. Anderson, Phys. Rev. 109, 1492 (1958).

[2] E. Abrahams, P.W. Anderson, D.C. Licciardello, T.V. Ramakrishnan, Phys. Rev. Lett. 42, 673 (1979).

[3] P.W. Anderson, Philos. Mag. B 52, 505 (1985).

[4] S. John, Phys. Rev. Lett. 58, 2486 (1987).

[5] A.Z. Genack, A.A. Chabanov, J. Phys A, Math. Gen. 38, 10465 (2005).

[6] D.S. Wiersma, P. Bartolini, A. Lagendijk, R. Righini, Nature (London) 390, 671 (1997).

[7] F. Scheffold, R. Lenke, R. Tweer, G. Maret, Nature (London) 398, 206 (1999).

[8] M. Störzer, P. Gross, Ch.M. Aegerter, G. Maret, Phys. Rev. Lett. 96, 063904 (2006).

[9] R.L. Weaver, Wave Motion 12, 129 (1990); Phys. Rev. B 47, 1077 (1993).

[10] A.A. Chabanov, M. Stoychev, A.Z. Genack, Nature (London) 404, 850 (2000).

[11] U. Kuhl, H.J. Stöckmann, Physica E 9, 384 (2001); P. Sebbah, B. Hu, J.M. Klosner, A.Z. Genack, Phys. Rev. Lett. 96, 183902 (2006); K.Yu. Bliokh, Yu.P. Bliokh, V. Freilikher, A.Z. Genack, B. Hu, P. Selbah, Phys. Rev. Lett. 97, 243904 (2006).

[12] R. Dalichaouch, J.P. Armstrong, S. Schultz, P.M. Platzman, S.L. McCall, Nature (London) 354, 53 (1991); A. Kudrolli, V. Kidambi, S. Sridhar, Phys. Rev. Lett. 75, 822 (1995); P. Pradhan, S. Sridhar, Phys. Rev. Lett. 85, 2360 (2000); H.-J. Stöckmann, M. Barth, U. Dörr, U. Kuhl, H. Schanze, Physica E 9, 571 (2001).

[13] J. Barthélemy, O. Legrand, F. Mortessagne, Phys. Rev. E 71, 016205 (2005).

[14] M.M. Sigalas, C.M. Soukoulis, C.-T. Chan, D. Turner, Phys. Rev. B 53, 8340 (1996).

[15] A. Derode, A. Tourin, M. Fink, Phys. Rev. E 64, 036605 (2001).

[16] H.C. Van de Hulst, Light Scattering by Small Particles, Dover Publications, New York 1981.

[17] B.C. Gupta, Z. Ye, Phys. Rev. E 67, 036606 (2003).

[18] J.S. Hersch, E.J. Heller, Phys. Rev. Lett. 81, 3059 (1998).

[19] P. Sebbah, C. Vanneste, Phys. Rev. B 66, 144202 (2002).

[20] J. Barthélemy, Ph.D. Thesis, Université Paris 7 - Denis Diderot, Paris 2003 (URL tel.ccsd.cnrs.fr/documents/archives0/00/00/41/14/).

[21] A. Gokirmak, D.-H. Wu, J.S.A. Bridgewater, S.M. Anlage, Rev. Sci. Instrum. 69, 3410 (1998).

[22] A. Taflove, Computational Electrodynamics: The Finite-Difference Time-Domain Method, Artech House, Norwood, MA 1995; the numerical code we use is directly inspired from the one developed by C. Vanneste in Ref. [19].

[23] J.P. Berenger, J. Comput. Phys. 114, 185 (1995).

[24] F.A. Pinheiro, M. Rusek, A. Orlowsky, B.A. van Tiggelen, Phys. Rev. E 69, 026605 (2004). 